\title{
Application of Chimera Grid Scheme to Combustor Flowfields at all Speeds
}

Shaye Yungster

Institute for Computational Mechanics in Propulsion

Cleveland, Ohio

Kuo-Huey Chen

The University of Toledo

Toledo, Ohio

January 1997

Prepared for

Lewis Research Center

Under Cooperative Agreement NCC3-370

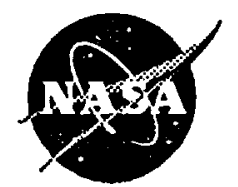

National Aeronautics and Space Administration

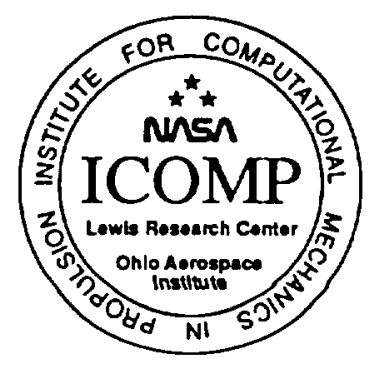





\title{
Application of Chimera Grid Scheme to Combustor Flowfields at all Speeds
}

\author{
Shaye Yungster \\ Institute for Computational Mechanics in Propulsion \\ NASA Lewis Research Center, Cleveland, Ohio \\ and \\ Kuo-Huey Chen* \\ The University of Toledo and \\ NASA Lewis Research Center, Cleveland, Ohio
}

\begin{abstract}
A CFD method for solving combustor flowfields at all speeds on complex configurations is presented. The approach is based on the ALLSPD-3D code which uses the compressible formulation of the flow equations including real gas effects, nonequilibrium chemistry and spray combustion. To facilitate the analysis of complex geometries, the chimera grid method is utilized. To the best of our knowledge, this is the first application of the chimera scheme to reacting flows. In order to evaluate the effectiveness of this numerical approach, several benchmark calculations of subsonic flows are presented. These include steady and unsteady flows, and bluff-body stabilized spray and premixed combustion flames. The results demonstrate the effectiveness of the combined ALLSPD$3 \mathrm{D} /$ chimera grid approach for analyzing subsonic combustor flowfields down to the incompressible limit.
\end{abstract}

\section{Introduction}

The numerical simulation of flow within a combustor requires a CFD code capable of solving the three-dimensional Navier-Stokes equations with finiterate chemistry on complex geometries. The compressible formulation is usually required since in many combustor applications velocities can range from low subsonic to supersonic. Even when the Mach number is low throughout the combustor, a compressible formulation is still necessary because of the wide density variation resulting from the combustion process. The computation of flowfields involving regions of low subsonic velocities using a compressible formulation is complicated by two main numerical difficulties. First, the flow equations become "stiff" at low Mach numbers. The degree of stiffness is determined by the ratio of the largest to the smallest eigenvalues. When the Mach number approaches zero, this ratio becomes infinitely large, and the Navier-Stokes equations become singular. In theory this difficulty can be circumvented by using implicit schemes. However, due to factorization errors, such schemes usually produce very poor convergence rates for problems involving very low Mach

*NASA Resident Research Associate at

Lewis Research Center. numbers. Second, the pressure gradient terms in the momentum equations become singular as the Mach number approaches zero. (In nondimensional variables, the pressure term is of order $1 / M^{2}$ while the convective term is of order unity). This singularity problem causes large roundoff errors at low Mach numbers.

At NASA Lewis Research Center a new CFD code, ALLSPD-3D ${ }^{1}$, is being developed for efficiently solving combustor flowfields at all speeds. This code solves the three-dimensional chemical nonequilibrium $N$ avier-Stokes equations over a wide range of Mach numbers. The method used is based on the strong conservation form of the governing equations, but utilizes primitive variables as unknowns. To overcome the difficulties for low Mach number calculations a preconditioning matrix is introduced $^{2}$. The singular behavior of the pressure gradient terms is circumvented by expressing the pressure as a sum of a reference pressure and a gauge pressure ${ }^{2}$, as discussed below. Real gas properties and nonequilibrium chemistry are considered. Turbulence is modeled with a low-Reynolds number $\kappa-\epsilon$ model developed by Shih et al. ${ }^{3}$, and a stochastic model is used to compute liquid spray combustion ${ }^{4}$.

In addition to having efficient numerics and accurate physical models, the computational method must be able to handle complex geometries. An efficient approach for this purpose is the domain decomposition method known as the chimera scheme ${ }^{5}$. This approach uses a composite of independent overlapping grids to discretize a complex geometrical configuration. The main advantages of the chimera method include: 1) simplification of the grid generation process by permitting a modular approach; 2) improvement in computational efficiency by providing better flowfield resolution with fewer grid points; 3 ) simplification in the application of boundary conditions; and 4) the possibility of allowing relative motion between system components without requiring a new grid to be generated at each time step.

Some of the disadvantages to the use of chimera grids are the complexity in the bookkeeping that tracks relationships among grids, and the fact that conservation is not strictly enforced at interface boundaries. In the present work we show that for low Mach number flows, mass conservation does not appear to present a serious problem.

The objective of this study is to evaluate the effectiveness of the combined ALLSPD-3D/chimera grid scheme approach for computing combustor flowfields. To the best of our knowledge, the chimera grid scheme has not been 
applied to reacting flows previously. The numerical formulation is described below, followed by a description of the numerical method and chimera scheme. Results are then presented for several benchmark tests including a channel flow and steady and unsteady flow over a bluffbody. Results are also presented for a bluff-body stabilized spray combustion flame.

\section{Mathematical Formulation}

The three-dimensional, unsteady, compressible, density-weighted time-averaged Navier-Stokes equations and species transport equations for a chemically reacting gas of $N$ species written in generalized nonorthogonal coordinates can be expressed as

$$
\begin{aligned}
& \boldsymbol{\Gamma} \frac{\partial \hat{\mathbf{Q}}}{\partial \tau^{*}}+\frac{\partial \tilde{\mathbf{Q}}}{\partial \tau}+\frac{\partial\left(\tilde{\mathbf{E}}-\tilde{\mathbf{E}}_{v}\right)}{\partial \xi}+\frac{\partial\left(\overline{\mathbf{F}}-\tilde{\mathbf{F}}_{v}\right)}{\partial \eta}+\frac{\partial\left(\overline{\mathbf{G}}-\tilde{\mathbf{G}}_{v}\right)}{\partial \zeta} \\
& =\tilde{\mathbf{H}}_{c}+\tilde{\mathbf{H}}_{l},
\end{aligned}
$$

where the vectors $\overline{\mathbf{Q}}, \overline{\mathbf{E}}, \overline{\mathbf{F}}, \overline{\mathbf{G}}, \overline{\mathbf{E}}_{v}, \overline{\mathbf{F}}_{v}, \overline{\mathbf{G}}_{v}, \overline{\mathbf{H}}_{c}$ and $\tilde{\mathbf{H}}_{\boldsymbol{l}}$ are defined as

$$
\begin{aligned}
\tilde{\mathbf{Q}} & =\frac{1}{J} \mathbf{Q} \\
\tilde{\mathbf{E}} & =\frac{1}{J}\left(\xi_{t} \mathbf{Q}+\xi_{x} \mathbf{E}+\xi_{y} \mathbf{F}+\xi_{z} \mathbf{G}\right) \\
\tilde{\mathbf{F}} & =\frac{1}{J}\left(\eta_{t} \mathbf{Q}+\eta_{x} \mathbf{E}+\eta_{y} \mathbf{F}+\eta_{z} \mathbf{G}\right) \\
\tilde{\mathbf{G}} & =\frac{1}{J}\left(\zeta_{t} \mathbf{Q}+\zeta_{x} \mathbf{E}+\zeta_{y} \mathbf{F}+\zeta_{z} \mathbf{G}\right) \\
\tilde{\mathbf{E}}_{v} & =\frac{1}{J}\left(\xi_{x} \mathbf{E}_{v}+\xi_{y} \mathbf{F}_{v}+\xi_{z} \mathbf{G}_{v}\right) \\
\tilde{\mathbf{F}}_{v} & =\frac{1}{J}\left(\eta_{x} \mathbf{E}_{v}+\eta_{y} \mathbf{F}_{v}+\eta_{z} \mathbf{G}_{v}\right) \\
\tilde{\mathbf{G}}_{v} & =\frac{1}{J}\left(\zeta_{x} \mathbf{E}_{v}+\zeta_{y} \mathbf{F}_{v}+\zeta_{z} \mathbf{G}_{v}\right) \\
\tilde{\mathbf{H}}_{c} & =\frac{1}{J} \mathbf{H}_{c} \\
\tilde{\mathbf{H}}_{l} & =\frac{1}{J} \mathbf{H}_{l}
\end{aligned}
$$

In the above expressions, $\tau, \xi, \eta$ and $\zeta$ are the temporal and spatial generalized coordinates and $\xi_{t}, \eta_{t}$ and $\zeta_{t}$ are the grid speed terms. $\xi_{x}, \xi_{y}, \xi_{z}, \eta_{x}, \eta_{y}, \eta_{z}, \zeta_{x}, \zeta_{y}$ and $\zeta_{z}$ are the metric terms and $J$ is the transformation Jacobian. The vectors $\mathbf{Q}, \mathbf{E}, \mathbf{F}, \mathbf{G}, \mathbf{E}_{v}, \mathbf{F}_{\boldsymbol{v}}$ and $\mathbf{G}_{\boldsymbol{v}}$ in the above definitions are

$$
\begin{aligned}
\mathbf{Q}= & \left(\rho, \rho u, \rho v, \rho w, \rho E, \rho \kappa, \rho \epsilon, \rho Y_{1}^{\prime}, \cdots, \rho Y_{N-1}\right)^{T} \\
\mathbf{E}= & \left(\rho u, \rho u^{2}+p, \rho u v, \rho u w,(\rho E+p) u, \rho u \kappa, \rho u \epsilon\right. \\
& \left.\rho u Y_{1}, \cdots, \rho u Y_{N-1}\right)^{T} \\
\mathbf{F}= & \left(\rho v, \rho u v, \rho v^{2}+p, \rho v w,(\rho E+p) v, \rho v \kappa, \rho v \epsilon,\right. \\
& \left.\rho v Y_{1}, \cdots, \rho v Y_{N-1}\right)^{T} \\
\mathbf{G}= & \left(\rho w, \rho u w, \rho v w, \rho w^{2}+p,(\rho E+p) w, \rho w \kappa\right. \\
& \left.\rho u \epsilon, \rho w Y_{1}, \cdots, \rho w Y_{N-1}\right)^{T}: \\
\mathbf{E}_{v}= & \left(0, \tau_{x x}, \tau_{x y}, \tau_{x z}, u \tau_{x x}+v \tau_{x y}+w \tau_{x z}+q_{x e}\right. \\
& \left.\tau_{x \kappa}, \tau_{x \epsilon}, q_{x 1}, \cdots, q_{x N-1}\right)^{T}
\end{aligned}
$$

$$
\begin{aligned}
\mathbf{F}_{v}= & \left(0, \tau_{y x}, \tau_{y y}, \tau_{y z}, u \tau_{y x}+v \tau_{y y}+w \tau_{y z}+q_{y_{e}},\right. \\
& \left.\tau_{y \kappa}, \tau_{y \epsilon}, q_{y 1}, \cdots, q_{y N-1}\right)^{T}, \\
\mathbf{G}_{v}= & \left(0, \tau_{z x}, \tau_{z y}, \tau_{z z}, u \tau_{z x}+v \tau_{z y}+w \tau_{z z}+q_{z e},\right. \\
& \left.\tau_{z \kappa}, \tau_{z \epsilon}, q_{z 1}, \cdots, q_{z N-1}\right)^{T},
\end{aligned}
$$

where $\rho, p, u, v, w, \kappa, \epsilon$ and $Y_{i}$ represent the density, pressure, Cartesian velocity components, turbulent kinetic energy, dissipation rate of turbulent kinetic energy and species mass fraction, respectively; $E=e+\frac{1}{2}\left(u^{2}+v^{2}+w^{2}\right)$ is the total internal energy with $e$ being the thermodynamic internal energy; The normal and shear stresses $\left(\tau_{x x}, \tau_{x y}, \ldots\right)$, energy $\left(q_{x_{e}}, \ldots\right)$, species $\left(q_{x_{i}}, \ldots\right)$, and turbulent diffusion $\left(\tau_{x \kappa}, \ldots\right)$ fluxes are given in $C$ hen and Shuen $^{4}$.

The first term in Eq. (1), $\Gamma \partial \hat{\mathbf{Q}} / \partial \tau^{*}$, is the time preconditioning term which is added to overcome the difficulties encountered in low Mach number calculations when the compressible formulation is used. The detailed theory about this specific treatment has been described in Shuen et al. ${ }^{2}$. The expressions in this term are

$$
\hat{\mathbf{Q}}=\frac{1}{J}\left(\begin{array}{c}
p_{g} \\
u \\
v \\
w \\
h \\
\kappa \\
\epsilon \\
Y_{1} \\
Y_{2} \\
\cdot \\
Y_{N-1}
\end{array}\right)
$$

$\Gamma=$

$$
\left[\begin{array}{llllllllllll}
1 / \beta & 0 & 0 & 0 & 0 & 0 & 0 & . & . & . & . & 0 \\
\mathbf{u} / \beta & \rho & 0 & 0 & 0 & 0 & 0 & . & . & . & . & 0 \\
\mathbf{v} / \beta & 0 & \rho & 0 & 0 & 0 & 0 & . & . & . & . & 0 \\
\mathbf{w} / \beta & 0 & 0 & \rho & 0 & 0 & 0 & . & . & . & . & 0 \\
\mathrm{H} / \beta-1 & \rho \mathrm{u} & \rho \mathbf{v} & \rho \mathrm{w} & \rho & 0 & 0 & . & . & . & . & 0 \\
\kappa / \beta & 0 & 0 & 0 & . & \rho & . & . & . & . & . & 0 \\
\boldsymbol{C} / \boldsymbol{\beta} & 0 & 0 & 0 & . & . & \rho & . & . & . & 0 & 0 \\
\mathrm{Y}_{1} / \beta & 0 & 0 & 0 & 0 & . & . & \rho & . & . & . & 0 \\
\mathrm{Y}_{2} / \beta & 0 & 0 & 0 & 0 & 0 & . & . & \rho & . & . & 0 \\
\cdot & . & . & . & . & . & 0 & . & . & \rho & . & 0 \\
. & . & . & . & . & . & . & 0 & . & . & \rho & 0 \\
\mathrm{Y}_{\mathrm{N}-1} / \beta & 0 & 0 & 0 & . & . & . & . & 0 & . & . & \rho
\end{array}\right]
$$

where $\hat{\mathbf{Q}}$ is the primitive dependent variable (to be solved), $\boldsymbol{\Gamma}$ is the preconditioning matrix, $\tau^{*}$ is the pseudotime and $h=e+{ }_{\rho}^{p}$ is the specific enthalpy of the gas mixture. $\beta$ is a scaling factor and is taken to be

$$
\beta=u^{2}+v^{2}+w^{2} \text {. }
$$

As described previously, this preconditioning technique allows us to calculate very low Mach number flows without difficulties. To circumvent the pressure gradient singularity problem, the pressure has been expressed as a 
sum of a constant reference pressure part, $p_{0}$, and a gauge pressure part, $p_{g}$ :

$$
p=p_{0}+p_{g}
$$

The reference pressure is usually selected as the freestream pressure. When this expression for the pressure is used in Eq. (1), the pressure gradient term in the nondimensional momentum equations becomes of order one as the Mach number approaches zero, and the singularity is eliminated from the equations.

The source term vectors $\mathbf{H}_{c}$ and $\mathbf{H}_{l}$ are

$$
\mathbf{H}_{c}=\left(\begin{array}{c}
0 \\
0 \\
0 \\
0 \\
0 \\
(\Psi-\rho \epsilon) \\
\left(c_{1} f_{1} \Psi-c_{2} f_{2} \rho \epsilon\right) \frac{\epsilon}{\kappa} \\
S_{1} \\
\cdot \\
S_{N-1}
\end{array}\right)
$$

and

$$
\mathbf{H}_{1}=\left(\begin{array}{c}
\sum_{p} n_{p} \dot{m}_{p} \\
\sum_{p} n_{p} \dot{m}_{p} u_{p}-\frac{4 \pi}{3} \rho_{p} r_{p}^{3} n_{p} \frac{d u_{p}}{d t} \\
\sum_{p} n_{p} \dot{m}_{p} v_{p}-\frac{4 \pi}{3} \rho_{p} r_{p}^{3} n_{p} \frac{d v_{p}}{d t} \\
\sum_{p} n_{p} \dot{m}_{p} w_{p}-\frac{4 \pi}{3} \rho_{p} r_{p}^{3} n_{p} \frac{d u_{p}}{d t} \\
\sum_{p} n_{p} \dot{m}_{p} h_{f s}-4 \pi r_{p}^{2} n_{p} h \Delta T \\
0 \\
0 \\
\sum_{p} n_{p} \dot{m}_{p} \\
0 \\
0 \\
0
\end{array}\right)
$$

The quantities related to the source term in the turbulent equations ( $\Psi, c_{1}$, etc.) are given by Chen and Shuen $^{4}$. The term $S_{i}$ is the chemical source term for species $i$, and is evaluated using standard methods (see for example Ref. 6). The spray source term $\mathbf{H}_{l}$, spray model, liquid-phase Lagrangian equations of motion and droplet mass and heat transfer equations are described in detail by Chen and Shuen ${ }^{4}$ and will not be repeated here. The temperature and density are calculated iteratively from the following equations

$$
\begin{gathered}
h_{i}=h_{f_{i}}^{0}+\int_{T_{\text {res }}}^{T} C_{p_{i}} d T, \\
p=\rho R_{u} T \sum_{i=1}^{N} \frac{Y_{i}}{W_{i}},
\end{gathered}
$$

where $R_{u}$ and $T_{\text {ref }}$ are the universal gas constant and reference temperature for thermodynamic properties, and $W_{i}, C_{p_{i}}, h_{i}, h_{f_{i}}^{\circ}$ are the molecular weight, constant pressure specific heat, thermodynamic enthalpy, and heat of formation of species $i$, respectively.
In reacting flow calculations, the evaluation of thermophysical properties is of vital importance. In this paper, the values of $C_{p i}, k_{l i}$, and $\mu_{l i}$ for each species are determined by fourth-order polynomials of temperature ${ }^{7}$. The specific heat of the gas mixture is obtained by mass concentration weighting of individual species. The thermal conductivity and viscosity of the mixture, however, are calculated using Wilke's mixing rule ${ }^{8}$. The binary mass diffusivity $D_{i j}$ between species $i$ and $j$ is obtained using the Chapman-Enskog theory ${ }^{8}$.

\section{Numerical Method}

\subsection{Iterative Scheme}

The gas-phase governing equations (Eq. (1)), which include the fluid dynamics, turbulence, and species conservation equations, could in principle be solved in a fully coupled manner. This approach would yield the best stability, robustness and convergence rate. However, such an approach requires large amounts of memory and expensive matrix inversions, which translates into more CPU time per iteration. This is specially true when many species are considered in the analysis. In order to compromise between the degree of coupling and an efficient use of computer resources a partially decoupled approach was adopted for solving Eq. (1). The governing equations are divided into three sets, i.e., five flow equations, two $\kappa-\epsilon$ turbulent equations and $N-1$ species equations as discussed in Ref. 1. Each individual set is solved in a coupled manner and iteratively.

The numerical scheme used in this study, and applied to each of the three sets of equations, is the LUSGS fully implicit method which is described in detail by Chen and Shuen ${ }^{1,4}$ and, for high-speed applications, by Yungster and Radhakrishnan ${ }^{9}$. The discretized form of Eq. (1) is expressed as follows.

$$
\begin{array}{r}
\left\{i _ { b } \left\{\mathbf{\Gamma}-\Delta \tau^{*}\left[\mathbf{S}+\mathbf{T}+\left(\frac{\partial \mathbf{A}}{\partial \xi}-\frac{\partial}{\partial \xi} \mathbf{R}_{\xi \xi \frac{\partial}{\partial \xi}}\right)\right.\right.\right. \\
\left.+\left(\frac{\delta \mathbf{B}}{\partial \eta}-\frac{\partial}{\partial \eta} \mathbf{R}_{\eta \eta} \frac{\partial}{\partial \eta}\right)+\left(\frac{\partial \mathbf{C}}{\partial \zeta}-\frac{\partial}{\partial \zeta} \mathbf{R}_{\zeta \zeta \frac{\partial}{\partial \zeta}}\right)\right] \\
\left.\left.+\mathbf{D}_{2}\right\}^{p}+\left(1-i_{b}\right) \mathbf{I}\right\} \Delta \hat{\mathbf{Q}}=\left\{-\Delta \tau^{*}(\mathbf{R})^{p}\right\} i_{b},
\end{array}
$$

where

$$
\begin{aligned}
& (\mathbf{R})^{p}=\frac{\partial\left(\overline{\mathbf{E}}-\overline{\mathbf{E}}_{\mathrm{v}}\right)}{\partial \xi}+\frac{\partial\left(\overline{\mathbf{F}}-\tilde{\mathbf{F}}_{\mathrm{v}}\right)}{\delta \eta}+ \\
& \frac{\partial\left(\tilde{\mathbf{G}}-\tilde{\mathbf{G}}_{\mathrm{v}}\right)}{\partial \zeta}-\overline{\mathbf{H}}_{c}-\tilde{\mathbf{H}}_{l}-\overline{\mathbf{D}}_{c}+\mathbf{D}_{\mathbf{4}}
\end{aligned}
$$

where the superscript $p$ denotes the previous iteration level, $\mathbf{S}$ and $\mathbf{T}$ are the Jacobian matrices for chemical and turbulent source terms, respectively, $\mathbf{A}, \mathbf{B}$ and $\mathbf{C}$ are the inviscid term Jacobians and $\mathbf{R}_{\xi \xi}, \mathbf{R}_{\eta \eta}$ and $\mathbf{R}_{\zeta \zeta}$ are the viscous term Jacobians. The expressions for these Jacobians are given in Chen et al $^{1}$. Note that the physical time term has not been included in the above expression, and therefore the solution marches forward in pseudotime. The second and forth order dissipation terms for LUSGS scheme are defined as

$$
\mathbf{D}_{2}=-0.5 \alpha\left[\frac{\partial^{2}}{\partial \xi^{2}}\left(\left|\lambda_{A}\right| \Gamma\right)+\frac{\partial^{3}}{\partial \eta^{2}}\left(\left|\lambda_{B}\right| \Gamma\right)+\frac{\partial^{2}}{\partial \zeta^{2}}\left(\left|\lambda_{C}\right| \Gamma\right)\right],
$$




$$
\mathbf{D}_{4}=\sigma \Gamma\left(\left|\lambda_{A}\right| \frac{\partial^{4} \hat{\mathbf{Q}}}{\partial \xi^{4}}+\left|\lambda_{B}\right| \frac{\partial^{4} \hat{\mathbf{Q}}}{\partial \eta}+\left|\lambda_{C}\right| \frac{\partial^{4} \hat{\mathbf{Q}}}{\partial \zeta^{+}}\right),
$$

where $\left|\lambda_{A}\right|,\left|\lambda_{B}\right|$ and $\left|\lambda_{C}\right|$ are the absolute value of the maximum eigenvalue in the $\xi, \eta$ and $\zeta$ directions, respectively. The $\overline{\mathbf{D}}_{c}$ in Eq. (3) represents a source term due to the partially decoupled procedure ${ }^{1}$. The $\alpha$ and $\sigma$ are defined in Chen and Shuen ${ }^{4}$. The term $i_{b}$ is a flag used with the chimera method which is described below.

\subsection{Chimera method}

In the chimera scheme approach, a system of relatively simple overlapping grids, each describing a component of a complex configuration, is combined into a composite grid to yield solutions for complex flow fields. A method to interconnect the grids is needed to determine when points of one grid fall within a body boundary of another (grid hole points), and to supply pointers so that one grid can provide data to update the boundary points of another. In this study, the code PEGSUS ${ }^{10}$ has been used for this purpose. The information is passed from one grid to another via trilinear interpolation. In order to distinguish the hole and grid boundary points from regular computational points, a blanking array, $i_{b}$, is used in the flow solver. For a hole or grid boundary point, $i_{b}$ is set to zero; otherwise it is set to one. In order to exclude the hole and grid boundary points from the solution procedure, all the terms in the right hand side and left hand side of equation (2) are multiplied by $i_{\delta}$, and the value of $\left(1-i_{b}\right) \mathbf{I}$ is added to the left hand side of this equation. Thus, for a regular computational point for which $i_{b}=1$, the normal scheme is maintained, but when $i_{b}=0$ the scheme reduces to $\Delta \hat{\mathbf{Q}}=0$ and thus $\hat{\mathbf{Q}}$ is not changed at a hole or boundary point. The solutions, $\hat{\mathbf{Q}}$, corresponding to the boundary points with $i_{b}=0$ are updated by the trilinear interpolation as described above.

\section{Results}

Results using the ALLSPD-3D/chimera approach are presented for four subsonic benchmark test cases: 1) nonreacting laminar channel flow; 2) nonreacting steady and unsteady bluff-body flows; 3 ) bluff-body stabilized spray combustion flame; and 4) bluff-body stabilized premixed combustion flame.

\subsection{Laminar Channel Flow}

The first case considers a low speed $(\mathrm{V}=0.051 \mathrm{~m} / \mathrm{sec})$, laminar flow in a channel with an arc bump. In order to test the numerical method, solutions are computed on a single zone grid $(41 \times 21 \times 17)$ and a 2 -block chimera grid with block dimensions of $(27 \times 21 \times 17)$ and $(20 \times 23 \times 19)$. Figure 1 shows the single zone and the 2-block chimera grids. For the chimera grid, the overlapped region between two grids is indicated in Fig. 1b. The boundary conditions are (see Fig. 1a): $\xi_{\min }$ : subsonic inflow; $\xi_{\max }$ : subsonic outflow; $\eta_{\min }$ : no-slip wall; $\eta_{\max }$ : symmetry; $\zeta_{\min }:$ symmetry; $\zeta_{\max }$ : no-slip wall.

The convergence history is presented in Fig. 2. It is noticed that the rate of convergence for the chimera grid is nearly as good as that for the single zone grid. A four order of magnitude reduction in the residual is obtained in approximately 1000 iterations. It is also worth pointing out that the overhead in memory requirements and CPU time due to the use of chimera grids is negligible.

The velocity magnitude along the center of the channel is plotted in Fig. 3. Excellent agreement between the two solutions is obtained.

\subsection{Laminar Steady and Unsteady Bluff-Body Flows}

In this case, a circular cylinder is placed inside the channel described in the previous section. Individual overlapping grids are generated for the channel and cylinder as shown in Fig. 4. Laminar results were obtained for two inflow velocities corresponding to Reynolds numbers (based on the cylinder diameter) of $R e_{d}=36.7$ and $R e_{d}=73.4$. It is known ${ }^{11}$ that for an unbound flow over a circular cylinder, the upper limit of Reynolds number for a steady-state solution is about 40 . In the present case, the flowfield around the cylinder is constrained by the channel walls, however, this is not expected to change dramatically the limiting Reynolds number for steady flow. Indeed, the results indicate that for the low Reynolds number case $\left(R e_{d}=36.7\right)$ the flow reaches a steady state, while for the $R e_{d}=73.4$ case the flow exhibits an unsteady, periodic vortex shedding behavior.

The steady-state solution for the $R e_{d}=36.7$ case is presented in Fig. 5 in the form of velocity vectors. One of the main concerns regarding the use of the chimera method is the fact that since interpolation is used to connect grids, conservation is not strictly enforced at the interface boundaries. To test the overall mass conservation, the mass flow rate across the various $\xi=$ const channel planes was computed for this case, and the results plotted in Fig. 6. The gap in the plot corresponds to the location occupied by the circular cylinder. This plot clearly indicates that the error due to interpolation is very small, less than $0.5 \%$.

Figure 7 shows a series of velocity vectors at selected pseudo-times during one of the periodic cycles of the $R e_{d}=73.4$ case. This figure illustrates the changing patterns of the wake. In Fig. 7f, the vortex structure behind the cylinder is nearly identical to that observed at the beginning of the cycle (Fig. 7a). The unsteady periodic nature of this flowfield can also be clearly observed in the convergence plot discussed later.

\subsection{Nonpremixed Bluff-Body Stabilized Flame}

The application of the present chimera grid method to combustion flow is demonstrated here using the same grid setup as the previous case. The circular cylinder is used as a flame holding device to stabilize the flame. For the present test purpose, the flow is assumed laminar and the Reynolds number based on the cylinder diameter and inlet air velocity is 57.7 . The inlet air temperature is 600 $K$. Liquid methanol was injected from 10 injection holes behind the cylinder. The schematic of this injection is depicted in Fig. 8. The liquid methanol was assumed to be fully atomized with the initial drop diameters ranging from 20 to $100 \mu \mathrm{m}$. Five species $\left(\mathrm{CH}_{3} \mathrm{OH}, \mathrm{O}_{2}, \mathrm{~N}_{2}, \mathrm{CO}_{2}\right.$, and $\mathrm{H}_{2} \mathrm{O}$ ) were considered in this calculation. A singlestep global reaction chemistry model reported in Westbrook and Dryer ${ }^{12}$ was used. The fuel/air ratio is 0.1 . The results of this calculation at three selected streamwise planes are presented in Fig. 9. Contour plots for fuel 
and oxidizer mass fractions are shown in Figs. 9a and $9 \mathrm{~b}$, and temperature contours are shown in Fig. 9c. Near the liquid injection, there is a relatively fuel-rich core region due to significant amount of evaporation of the liquid methanol. This can be clearly seen in the fuel mass fraction contours. The flame was established along the outer edge of this fuel-rich core region where gaseous fuel and oxidizer are well mixed. The first temperature contour plane behind the cylinder indicates this flame pattern. The second contour plane indicates that at this station all the fuel has been consumed, and a nearly circular high temperature region is established. Further downstream, the surrounding air mixes with the combustion products reducing the temperature levels significantly.

For the previous three bluff-body non-reacting and reacting flows, the convergence histories are shown in Fig. 10. For non-reacting flows, steady state convergence is observed for the low Reynolds case $\left(R e_{d}=36.7\right)$, while a periodic convergence pattern exhibits for the higher Reynolds number flow $\left(R e_{d}=73.4\right)$. This periodic convergence pattern is consistent with the vortex shedding flow previously discussed. For the combustion convergence curve, a spike near 100 iterations indicates the time of ignition. Several small peaks near the end of the convergence curve are due to the numerical treatment of the spray source terms that are updated every 20 iterations in the present calculation.

\subsection{Premixed Bluff-Body Stabilized Flame}

To validate the current ALLSPD-3D/chimera method on the accuracy of the combustion flow predictions, a premixed propane flame with available experimental and numerical data ${ }^{13}$ was chosen in this study. The test case consists of a straight channel with a rectangular cross section and a wedge flame holder. Although the experimental test was conducted in a fully three-dimensional rig, a two-dimensional flow is calculated along the symmetry plane where the experimental data and other numerical predictions are available to be compared with. A schematic, taken from Ref. 13, of the experimental test setup is shown in Fig. 11. The gaseous propane fuel is premixed with air before entering the combustion section. The inlet temperature and velocity are $288 \mathrm{~K}$ and $17.06 \mathrm{~m} / \mathrm{s}$, respectively. The equivalence ratio is 0.61 . Five species $\left(\mathrm{C}_{3} \mathrm{H}_{8}, \mathrm{O}_{2}, \mathrm{~N}_{2}, \mathrm{CO}_{2}\right.$, and $\left.\mathrm{H}_{2} \mathrm{O}\right)$ were considered in this calculation. A single-step global reaction chemistry model reported in Westbrook and Dryer ${ }^{12}$ was used.

Two grids were used in this study. Figure 12 shows a Cartesian grid $(175 \times 41)$ for the channel and an O-type grid $(31 \times 130)$ around the wedge flame holder. Since the combustor is symmetric in the $y$-direction, only half of the domain was actually calculated. The converged results are shown in Fig. 13. The temperature contours are presented in Fig. 13a. The combustion flame is stablized behind the flame holder with a vivid flame front that starts near the tip of the flame holder and extends further downstream. Figures $13 \mathrm{~b}$ and $13 \mathrm{c}$ show the comparison for temperature profiles between the current predictions and two other data at two streamwise stations behind the flame holder. The first station is located 0.15 meter behind the flame holder and the second station is at 0.35 meter. The temperature profile at the 0.15 meter station compares favorably with the numerical predictions and experimental data reported in Ref. 13. However, both current results and the other numerical predictions at the 0.35 meter station do not agree that well with the experimental data. As indicated in Ref. 13, the diffusion/mixing rate is too slow compared to the experimental data. This can be seen in both temperature profiles. The isotropic assumption of the current low Reynolds turbulence model may not be adequate in predicting the non-isotropic turbulence in the shear layer region close to the flame holder. Although many issues exist and further study is needed to improve the accuracy of the current simulation, the use of the chimera method presented no difficulty in obtaining reasonably good results

\section{Conclusions}

The successful implementation of the chimera grid scheme into the ALLSPD-3D code has extended its capability to handle complex configurations. The use of the chimera method for domain decomposition resulted in very low overhead in CPU time and memory requirements, and did not degrade the excellent convergence characteristics of the numerical method. The effectiveness of this numerical approach was demonstrated by computing several subsonic, nonreacting, steady and unsteady flows, and bluff-body stabilized spray and premixed combustion flames. Mass conservation error was shown to be very small $(<0.5 \%)$ for these cases. Also, the large gradients in the flow variables (i.e., temperature, density, species concentrations, etc) resulting from combustion processes did not have a negative impact on the interpolation process. The results of this work indicate that the combined ALLSPD-3D/chimera grid approach is a powerful tool for computing subsonic combustor flowfields down to the incompressible limit.

\section{Acknowledgments}

The authors wish to thank R.W. Tramel and N.E. Suhs of Calspan Corporation/AEDC Operations and P. Bunning of NASA Ames Research Center for providing us with the PEGSUS code. The authors also thank Dr. K.$\mathrm{H}$. Kao of ICOMP for his help during the implementation of the chimera method.

\section{References}

1. Chen, K.-H. Shuen, J.-S. and Mularz, E. "A Comprehensive Study of Numerical Algorithms for Three-Dimensional, Turbulent, Non-Equilibrium Viscous Flows," AIAA Paper 95-0800, $1995^{1}$

2. Shuen, J.-S. Chen, K.-H. and Choi, Y., "A Coupled Implicit Method for Chemical Non-Equilibrium Flows at All Speeds," J. Comput. Phys., 106, 306 (1993).

3. Shih, T.H. and Lumley, J., "Kolmogorov Behavior of Near-Wall Turbulence and its application in Turbulence Modeling," Comp. Fluid Dyn., 1, 43, 1993.

4. Chen, K.-H. and Shuen, J.-S. " "Three-Dimensional Coupled Implicit Method For Spray Combustion Flows At All Speeds," AIAA 94-3047, 1994.

5. Steger, J. L. Dougherty, F. C. and Benek, J. A. "A Chimera Grid Scheme," in Advances in Grid Generation, K. N. Ghia and U. Chia, eds., ASME FED-5, 59-69, (1983).

\footnotetext{
${ }^{1}$ Information about the ALLSPD-3D code can also be obtained on the World Wide Web at: http://www.lerc.nasa.gov/Other_Groups/IFMD/allspd/
} 
6. Yungster, S. and Rabinowitz, M.J., "Computation of Shock-Induced Combustion Using a Detailed MethaneAir Mechanism," J. of Prop. and Power, Vol. 10, No. 4, July-Aug. 1994.

7. Gordon, S. and McBride, B.J., "Computer Program for Calculation of Complex Chemical Equilibrium Compositions, Rocket Performance, Incident and Reflected Shocks, and Chapman-Jouguet Detonations," NASA SP273, March 1976.

8. Reid, R. C. Prausniz, J. M. and Poling, B. E. The Properties of Gases and Liquids, fourth Ed., McGraw-Hill Publishing Co., N.Y., 1988.

9. Yungster, S. and Radhakrishnan, K., "A Fully Implicit Time-Accurate Method for Hypersonic Combustion: Application to Shock-Induced Combustion Instability," NASA TM 106707, Aug. 1994.

10. Benek, J. A. Steger, J. L. Dougherty, F. C. and Bunning, P. G. "Chimera: A Grid Embedding Technique" AEDC-TR-85-64, Arnold Air Force Station, TN, (1986).

11. Fornberg, B., "A Numerical Study of Steady Viscous Flow Past a Circular Cylinder," J. Fluid Mech., 98, $819(1980)$

12. Westbrook, C. K. and Dryer, F. L. "Simplified Reaction Mechanisms for the Oxidation of Hydrocarbon Fuels in Flames," Combustion Science and Technology, 27, 31 (1981).

13. Olovsson, S. "Combustion Calculations on a Premixed System with a Bluff Body Flameholder," AIAA 92-3470, 1992. 


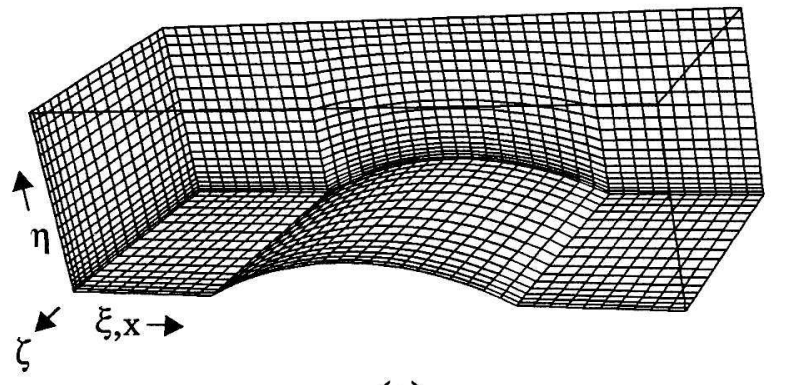

(a)

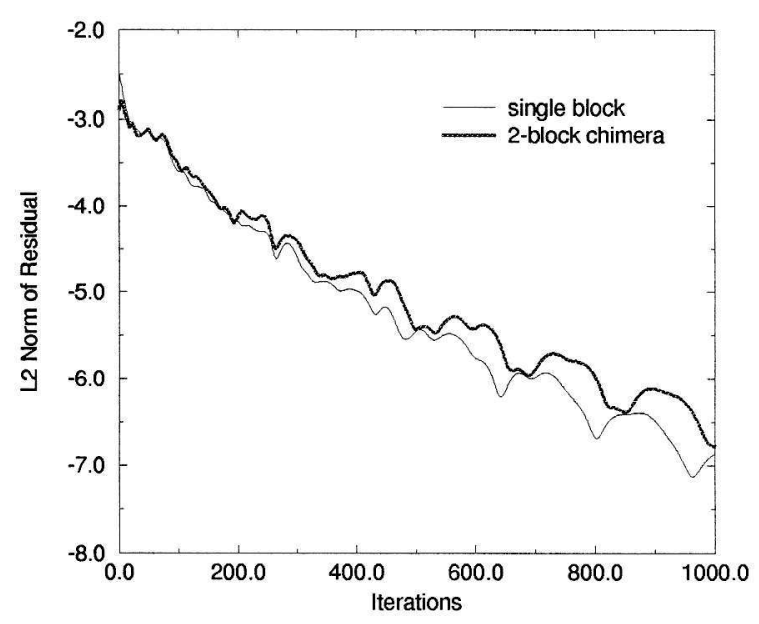

Figure 2. Convergence history

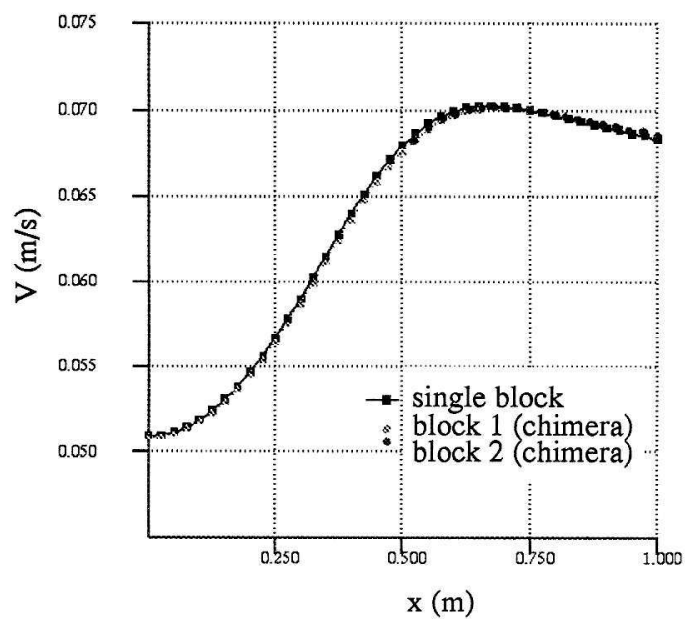

Figure 3. Comparison of velocity magnitude for single zone and chimera grids along channel centerline. 

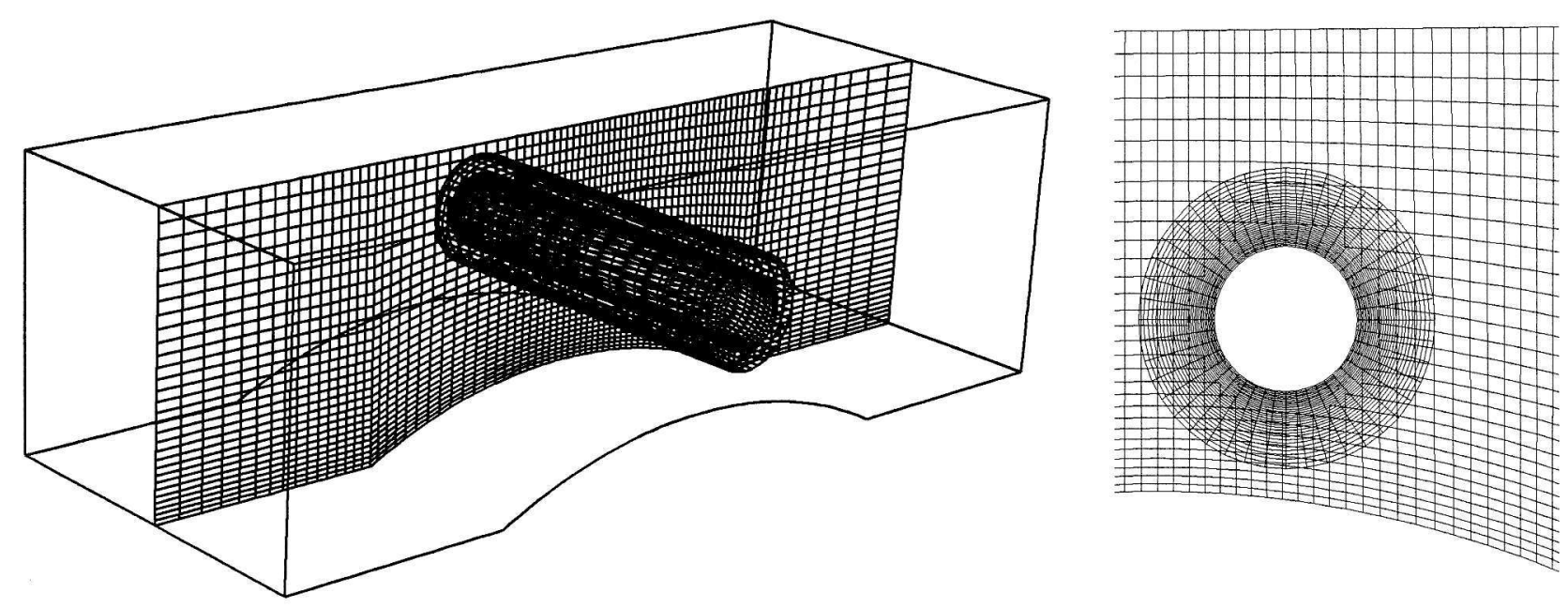

Figure 4. Two-zone chimera grid for channel/cylinder problem.

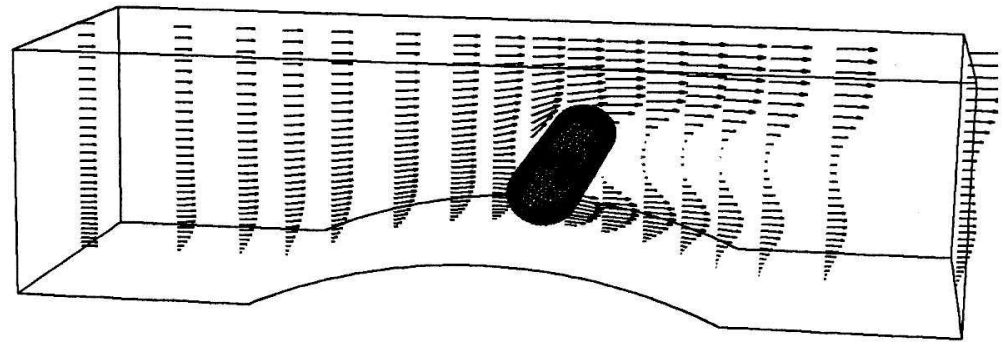

Figure 5. Computed velocity vectors. Steady flow $\left(\mathrm{Re}_{\mathrm{d}}=36.7\right)$

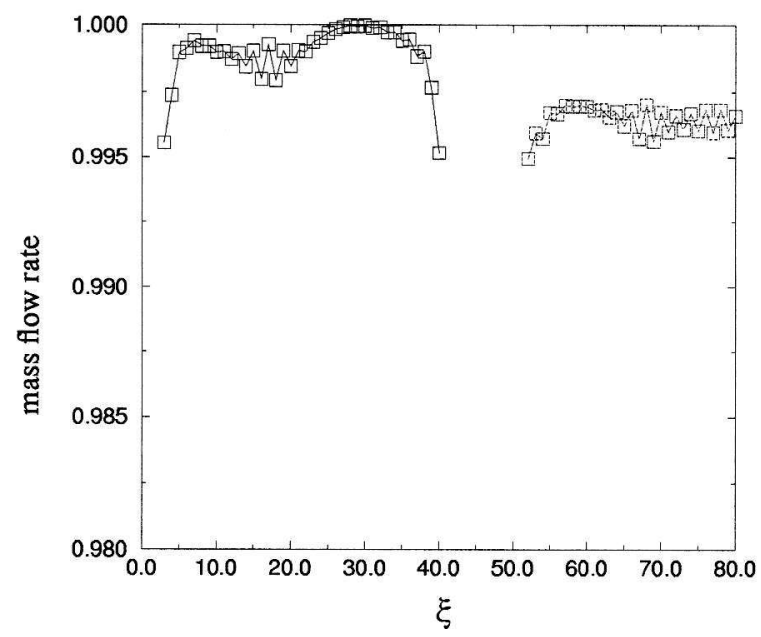

Figure 6. Normalized mass flow rate along the channel. $\left(\operatorname{Re}_{\mathrm{d}}=36.7\right)$ 


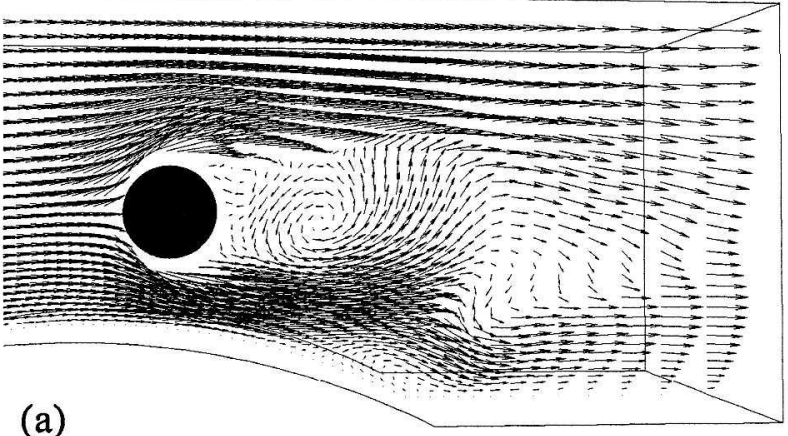

(a)

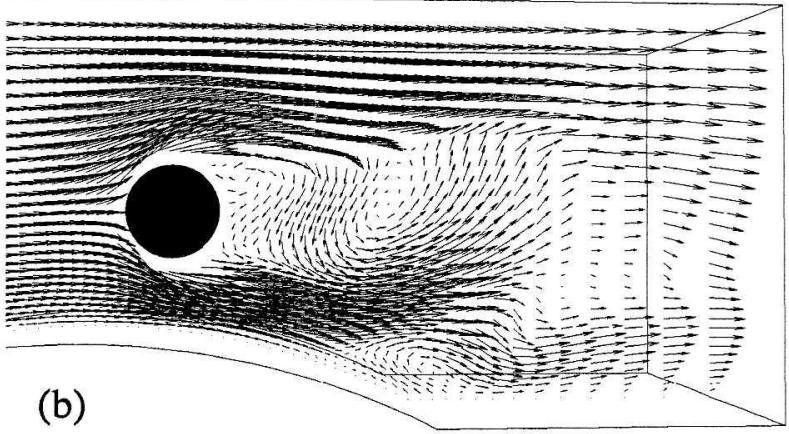

(b)

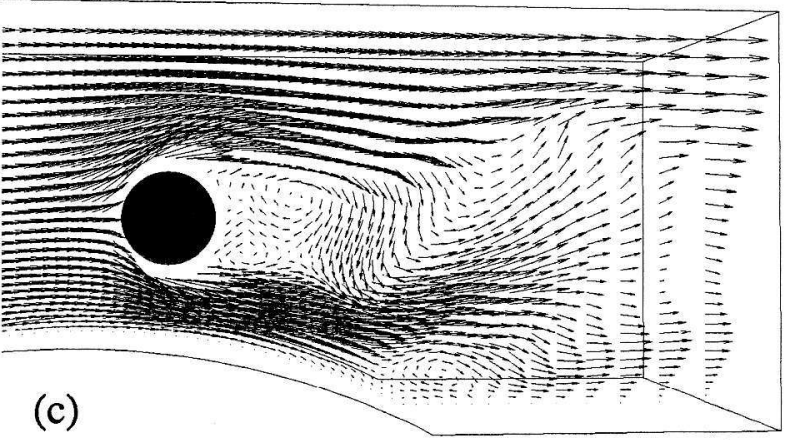

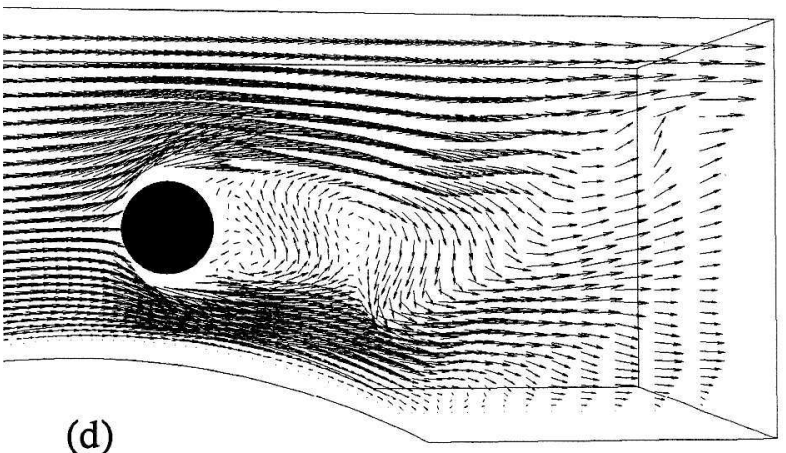
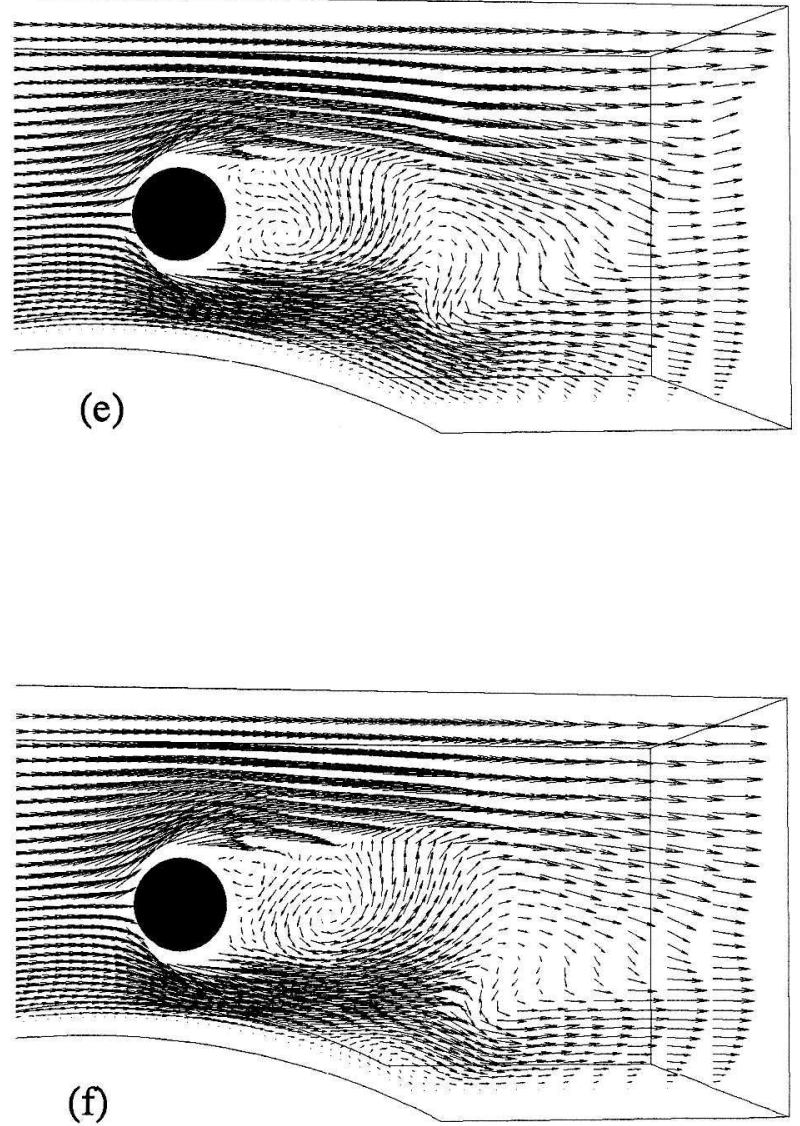

(f)

Figure 7. Velocity vector plots at six different times during one cycle. Unsteady flow $\left(\operatorname{Re}_{\mathrm{d}}=73.4\right)$. 

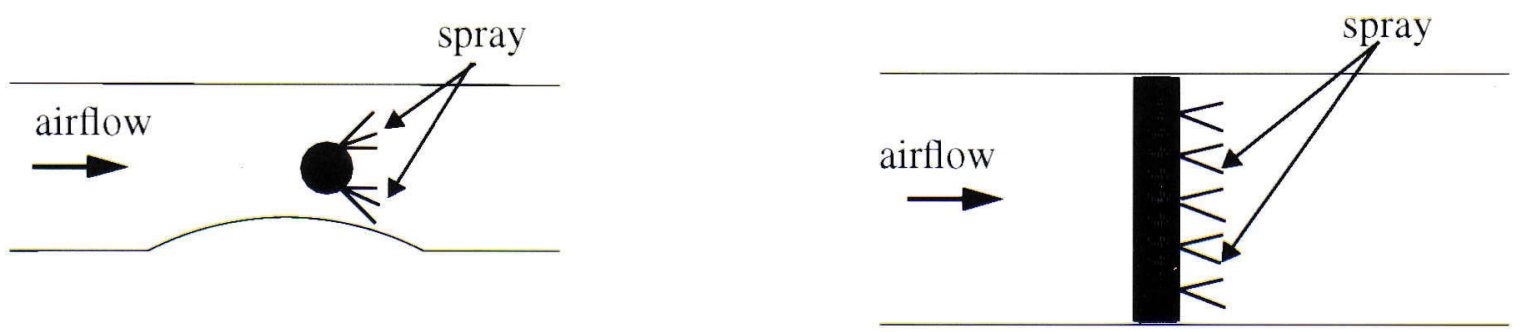

Figure 8. Schematic of liquid methanol spray injection.
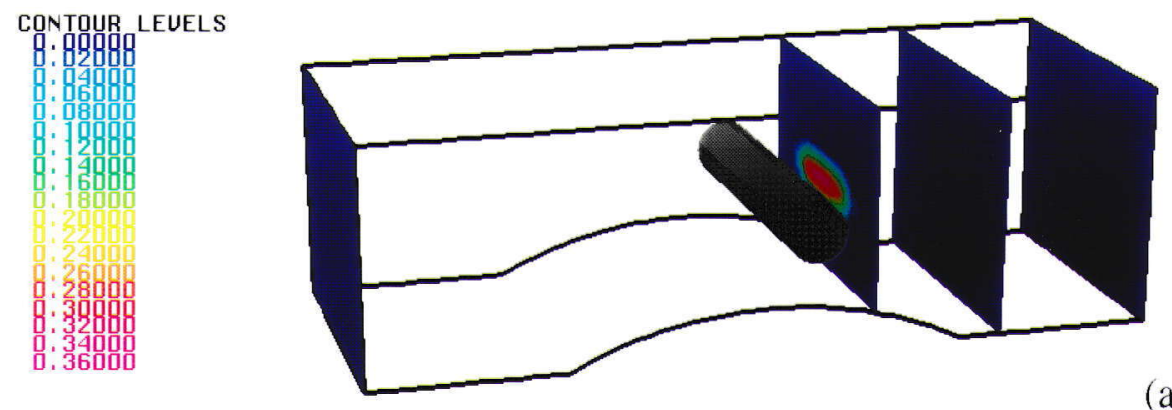

(a)
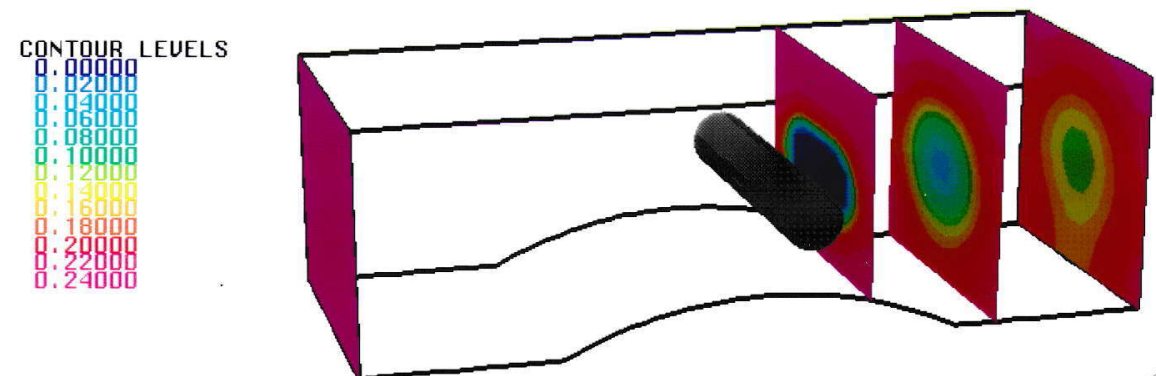

(b)
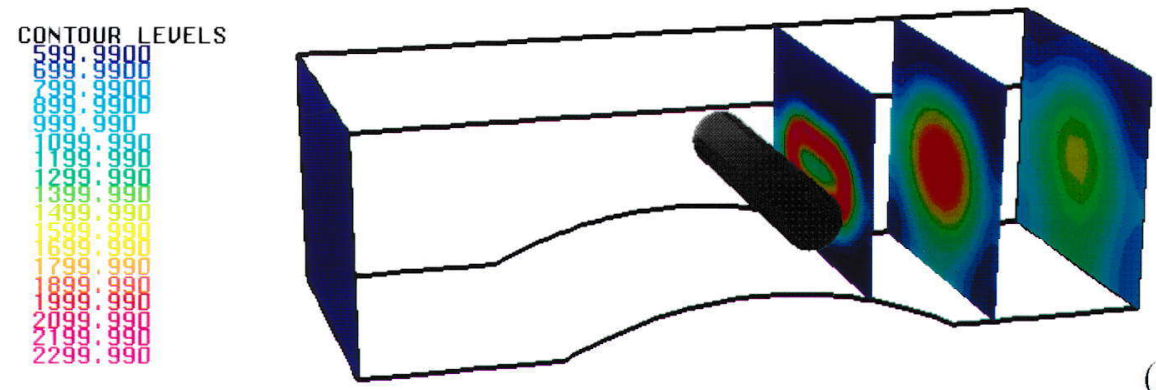

(c)

Figure 9. Spray combustion flowfield; (a) methanol mass fraction; (b) oxygen mass fraction; (c) temperature $(\mathrm{K})$. 


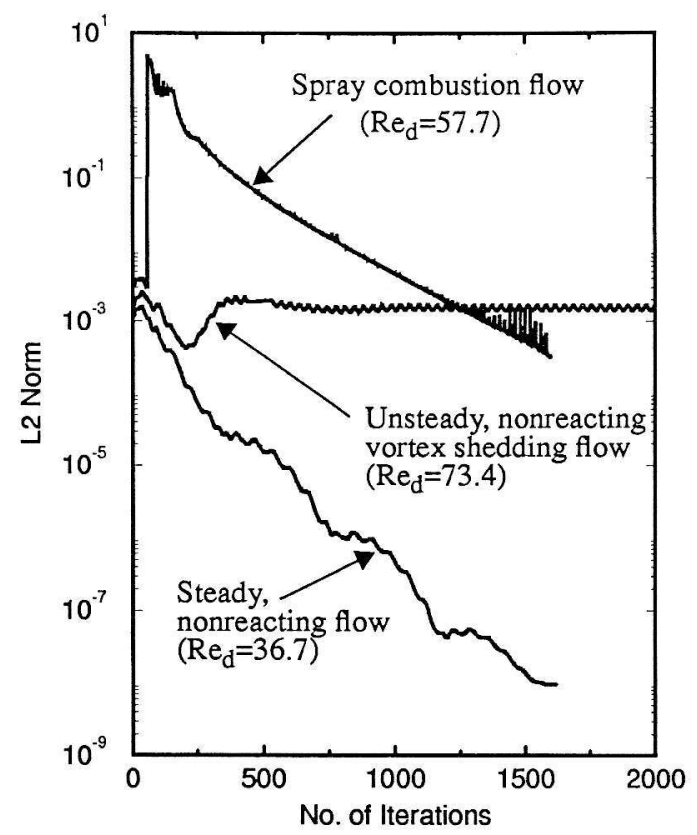

Figure 10. Convergence history

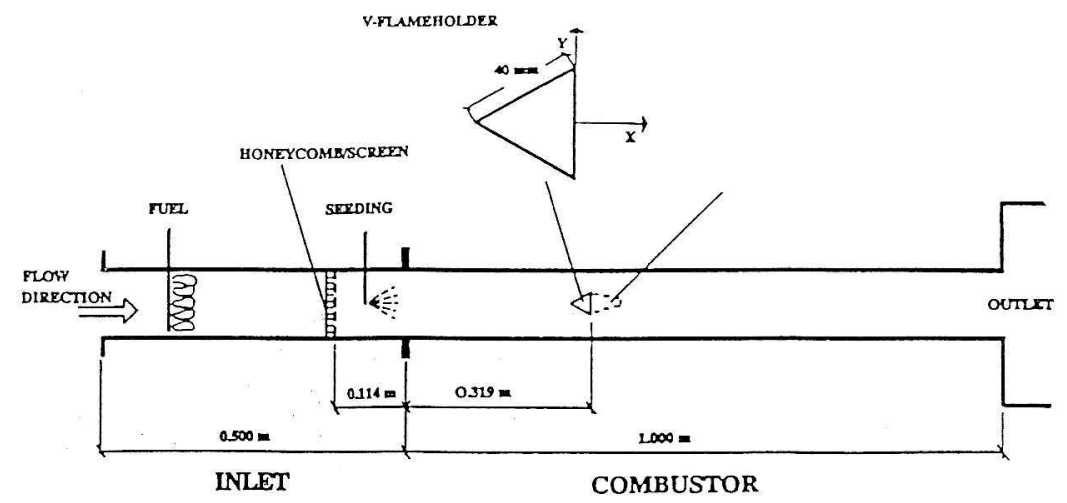

Figure 11. Schematic of premixed combustion experiment with bluff body flameholder (Ref. 13).

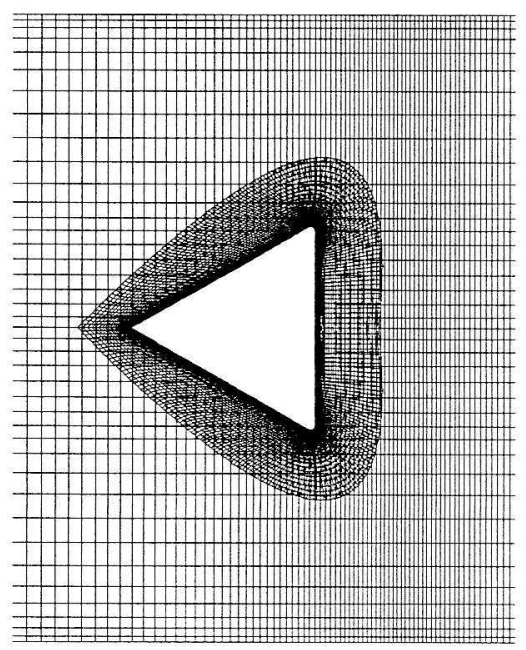

Figure 12. Two-zone chimera grid. 


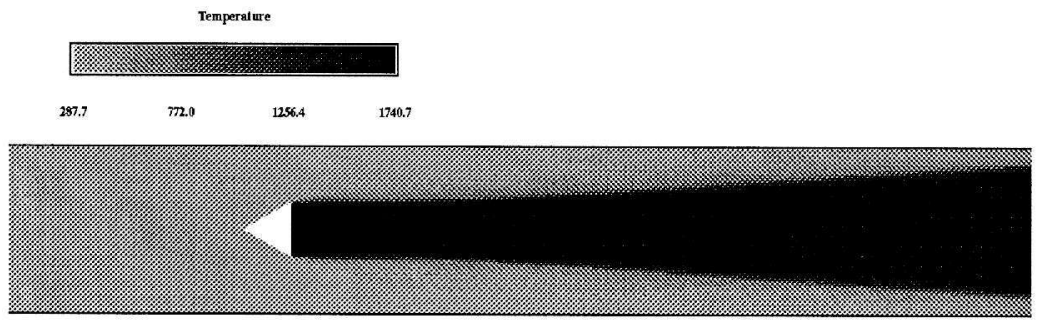

(a)
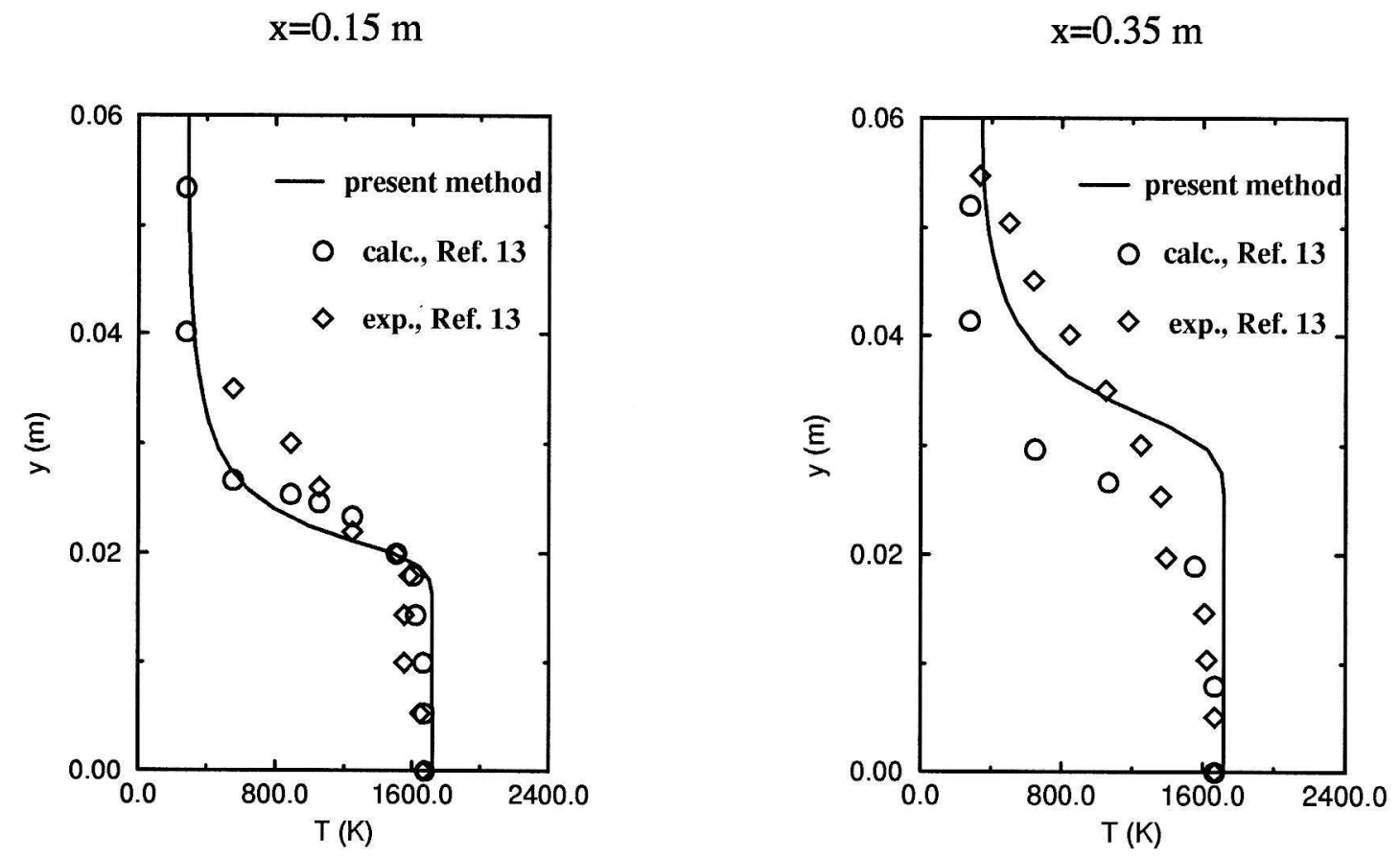

(b)

Figure 13. (a) Temperature contours and (b) temperature distribution along the $x=0.15 \mathrm{~m}$ and $\mathrm{x}=0.35 \mathrm{~m}$ stations for the premixed combustor. 

Public reporting burden for this collecton of hiomation L gathering and maintaining the data needed, and completing and reviewing the collection of informatlon. Send commente regarding this burden eatimate of any other anpect of this

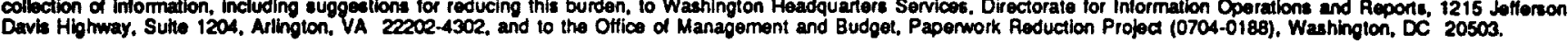

\begin{tabular}{|l|l|l|}
\hline 1. AGENCY USE ONLY (Leave blank) & 2. REPORT DATE & 3. REPOAT TYPE AND DATES COVERED
\end{tabular}

4. TITLE AND SUBTITLE

January 1997

Application of Chimera Grid Scheme to Combustor Flowfields at all Speeds

6. AUTHOA(S)

Shaye Yungster and Kuo-Huey Chen

7. PERFORMING ORGANIZATION NAME(S) AND ADDRESS(ES)

Institute for Computational Mechanics in Propulsion

22800 Cedar Point Road

Cleveland, Ohio 44142
Contractor Report

5. FUNDING NUMBERS

WU-505-90-5K

NCC3-370

8. PERFORMING ORGANIZATION REPORT NUMBER

E-10638

9. SPONSORINGMONITORING AGENCY NAME(S) AND ADDRESS(ES)

10. SPONSORING/MONITORING

AGENCY REPORT NUMBER

National Aeronautics and Space Administration

Lewis Research Center

Cleveland, Ohio 44135-3191

NASA CR-202321

ICOMP-97-02

\section{SUPPLEMENTARY NOTES}

Prepared for the 34th Aerospace Sciences Meeting and Exhibit sponsored by the American Institute of Aeronautics and Astronautics,

Reno. Nevada, January 15-18, 1996. Shaye Yungster, Institute for Computation Mechanics in Propulsion, NASA Lewis Research Center (work funded under NASA Cooperative Agreement NCC3-370); Kuo-Huey Chen, The University of Toledo. Toledo, Ohio 43606-3328. ICOMP Program Director, Louis A. Povinelli, organization code 5800. (216) 433-5818.

12. DISTAIBUTIONAVAILABILTTY STATEMENT

12b. DISTRIBUTION COOE

Unclassified -Unlimited

Subject Category 34

This publication is available from the NASA Center for AeroSpace Information, (301) 621-0390.

13. ABSTRACT (Maximum 200 words)

A CFD method for solving combustor flowfields at all speeds on complex configurations is presented. The approach is based on the ALLSPD-3D code which uses the compressible formulation of the flow equations including real gas effects, nonequilibrium chemistry and spray combustion. To facilitate the analysis of complex geometries, the chimera grid method is utilized. To the best of our knowledge, this is the first application of the chimera scheme to reacting flows. In order to evaluate the effectiveness of this numerical approach, several benchmark calculations of subsonic flows are presented. These include steady and unsteady flows, and bluff-body stabilized spray and premixed combustion flames. The results demonstrate the effectiveness of the combined ALLSPD-3D/chimera grid approach for analyzing subsonic combustor flowfields down to the incompressible limit.

\begin{tabular}{|c|c|c|c|}
\hline \multicolumn{3}{|c|}{ Preconditioning; Combustion; Overset grid; Spray } & $\begin{array}{l}\text { 15. NUMBER OF PAGES } \\
14\end{array}$ \\
\hline
\end{tabular}

NSN 7540-01-280-5500 